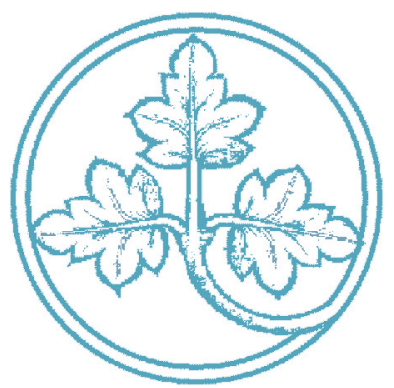

REITs ante portas Die Anpassung des deutschen Rechts an institutionelle Investoren in den Grundstücksund Mietmärkten

Christoph Engel

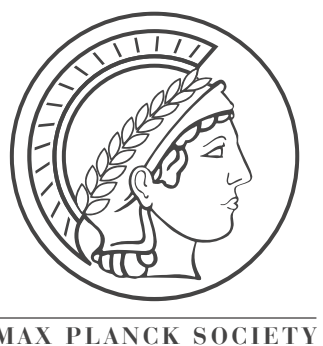




\section{REITs ante portas \\ Die Anpassung des deutschen Rechts an institutionelle Investoren in den Grundstücks- und Mietmärkten}

Christoph Engel

April 2008 


\title{
REITs ante portas \\ Die Anpassung des deutschen Rechts an institutionelle Investoren in den Grundstücks- und Mietmärkten
}

\author{
Christoph Engel
}

Wenn man einmal im Leben einen Ehering braucht, lernt man nicht Goldschmied. Man geht zum Spezialisten und profitiert von dessen Erfahrung, Geschick und Marktkenntnis. Wenn man einmal im Leben ein Haus braucht, lernt man Eigentümer. Man studiert den Markt, verhandelt die Finanzierung und wird sein eigener Hausmeister. Muss das so sein? In den USA und im Vereinigten Königreich ist es immer häufiger anders ${ }^{1}$. Immer mehr Grundstücke gehören institutionellen Investoren, insbesondere sog. REITs. Das gilt gerade auch für Wohnraum. Nun treten institutionelle Investoren auch in die deutschen Grundstücksmärkte ein (1). Das hat nicht nur Folgen für das Grundstücksrecht selbst (2), sondern auch für das Mietrecht (3) und das Bauplanungsrecht (4). Vor allem hat der Gesetzgeber die Entwicklung aber durch die Gestaltung der Rahmenbedingungen weitgehend in der Hand (5).

\section{Institutionelle Investoren auf deutschen Grundstücksmärkten}

Der Gesetzgeber hat den deutschen Rechtsraum gerade ausdrücklich für eine weitere Klasse institutioneller Investoren an den Grundstücksmärkten geöffnet ${ }^{2}$. Bislang spielen Real Estate Investment Trusts, oder kurz REITs ${ }^{3}$, in Deutschland praktisch keine Rolle. Ein REIT ist eine an der Börse notierte Aktiengesellschaft, die sich auf das Halten von Grundeigentum spezialisiert. Die Marktkapitalisierung der Anlage von REIT-Vermögen in deutschen Grundstücken betrug im Jahre 2002748 Mio \$. Das sind nur 0,26 \% des Weltmarktes. Zum Vergleich: die Kapitalisierung englischer Anlagen betrug zur gleichen Zeit 30.265 Mio \$. US-amerikanische Anlagen kamen sogar auf 149.771 Mio \$4. In der Vergangenheit sind REITs rasant gewachsen. Die weltweite Marktkapitalisierung hat sich zwischen 1972 und 2002 um den Faktor 86 vermehrt ${ }^{5}$. Schon

* Martin Hellwig danke ich für seine Anmerkungen zu einer früheren Fassung.

1 Ulrich Schacht und Katrin Gänsler, REITs in Deutschland und Großbritannien - ein Vergleich, in: Internationales Steuerrecht (2007) 99-106. Auch in diesen Ländern gibt es aber nach wie vor viel privates Wohungseigentum. Im Median hielt im Jahre 2004 jeder amerikanische Haushalt 160.000 \$ selbstgenutztes Wohnungseigentum, gegenüber 93.800 \$ Finanzeigentum,

http://www.federalreserve.gov/pubs/bulletin/2006/financesurvey.pdf.

2 Gesetz über deutsche Immobilien-Aktiengesellschaften mit börsennotierten Anteilen (REITG) v. 28.5.2007, BGBl. I 914.

3 Zum Hintergrund Dennis R. Capozza und Paul J. Seguin, Real Estate Investment Trusts, in: Real Estate Economics 31 (2003) 305-311; Su Han Chan, John Erickson und Ko Wang, Real Estate Investment Trusts. Structure, Performance, and Investment Opportunities. Oxford (2003).

4 A. Shaun Bond, G. Andrew Karolyi und Anthony B Sanders, International Real Estate Returns. A Multifactor, Multicountry Approach, in: Real Estate Economics 31 (2003) 481-500, 487.

5 Capozza und Seguin (FN 3) 305 f. 
diese Zahlen lassen auch für Deutschland erhebliche Änderungen erwarten. Im Mutterland der REITs, in den USA, haben sie sich immer stärker weg von einem Fonds und hin zu operativen Gesellschaften entwickelt, deren Anteile an der Börse gehandelt werden ${ }^{6}$. Sie sind immer größer und immer liquider geworden ${ }^{7}$. Sie spezialisieren sich immer enger auf bestimmte Typen von Grundstückseigentum ${ }^{8}$.

Die neuen REITs dürfen in Deutschland mit erheblicher Nachfrage rechnen. Ein wichtiger Nachfrager werden die Kommunen sein. Eine Studie hat 200 Kommunen befragt. Unter ihnen waren 34 Großstädte. 40\% der Befragten beabsichtigten, ihre Wohnungsbestände ganz oder teilweise zu veräußern. 54\% wollten hierdurch einen Sanierungsstau auflösen, 51\% Schulden abbauen ${ }^{9}$.

Die Zulassung von REITs ist allerdings nur der sichtbarste Ausdruck einer Entwicklung, die in anderen Rechtsformen schon früher eingesetzt hat. Ende Februar 2008 hielten offene Immobilienfonds ein Vermögen von 85.670 Mio €. Zum Vergleich: Aktienfonds hielten 206.997 Mio €, und Rentenfonds 180.385 Mio $€$. Der Anteil von offenen Immobilienfonds am gesamten Vermögen deutscher Publikums- und Spezialfonds betrug 6,1 \% ${ }^{10}$. Hinzu treten geschlossene Immobilienfonds, meist in der Rechtsform einer Kommanditgesellschaft. Da sie nicht zur Publizität verpflichtet sind, gibt es nur Schätzungen über das Anlagevolumen. Eine Schätzung für das Jahr 2003 geht von einem Volumen von ungefähr 6.000 Mio $€$ aus ${ }^{11}$. Schließlich sind ausländische Private Equity Gesellschaften am deutschen Immobilienmarkt engagiert. Im Gegensatz zu den Immobilienfonds halten sie auch Wohnimmobilien. In den Jahren 2001 bis 2006 haben solche Gesellschaften Wohnungen im Wert von 20.000 Mio $€$ gekauft $^{12}$.

Der normative Blick sollte deshalb weniger auf REITs als solche fallen. Wichtiger ist die generelle Veränderung der Eigentumsstruktur. Die Zulassung von REITs wird die Stellung institutioneller Anleger an den Grundstücksmärkten aber weiter verstärken. In ähnlicher Weise wie Immobilienfonds sind sie steuerlich privilegiert ${ }^{13}$. Ihre Anteile können an der Börse gehandelt werden. Das wird zusätzliches Kapital verfügbar machen. Nach ausländischen Erfahrungen ist damit zu rechnen, dass die REITs sich auf relativ enge Marktsegmente spezialisieren.

6 Su Han Chan, Wai-Kin Leung und Ko Wang, Changes in REIT Structure and Stock Performance. Evidence from the Monday Stock Anomaly, in: Real Estate Economics 33 (2005) 89-120, 89 f.

Ebd, 90 m.w.N.

Ebd.

Guido Eusani, Regierungsentwurf zum Real Estate Investment Trust-Gesetz (REIT-Gesetz) - Nur eine ,kleine“ Lösung, in: Neue Zeitschrift für Mietrecht (2007) 66-75, 71.

10 http://www.bvi.de/de/presse/pressemitteilungen/presse2008/pm010408_absatzstatistik/pm010408.pdf

11 http://de.wikipedia.org/wiki/Geschlossene_Fonds\#Geschlossene_Immobilienfonds.

12 FAZ 4.8.2006, 15; einen Überblick über den Wohnungsbesitz von Anlagegesellschaften in Deutschland gibt Stefan Kofner, Wohnimmobilien-Aktiengesellschaften, in: Wohnungswirtschaft und Mietrecht (2008) 68-72.

S. dazu unten 5 . 


\section{Folgen für das Grundstücksrecht}

Je stärker sich institutionelle Investoren am deutschen Immobilienmarkt etablieren, desto stärker wird das Vorhalten von Grundstücken, Gebäuden und Wohnraum zu einem selbstständigen Beruf. Man kann auch formulieren: wie im späten 19. Jahrhundert gibt es wieder „Rentiers“, nur dass sie ganz anders verfasst sind. Dadurch wird mehr Kapital zur Anlage am Grundstücksmarkt verfügbar. Weil der Markt wächst, werden Objekte finanzierbar, die andernfalls nicht entstanden wären. Die Allokation verbessert sich. Das in Grundstücken steckende Kapital der Volkswirtschaft wird produktiver genutzt.

Weil sich die Grundstücksmärkte durch professionelle Anleger radikal ändern, muss das Grundstücksrecht neu durchdacht werden. Einzelne Regeln mögen an Bedeutung verlieren. Wenn ein institutioneller Anleger ein paar Grundstücke verkauft, ist das nur ein Bruchteil seines Vermögens. Zum Schutz seiner Eigentümer ist nicht die Kontrolle der einzelnen Transaktionen erforderlich. Es kommt vielmehr darauf an, dass sich die Risikostruktur nicht unvermittelt ändert. Aus der Sicht der Anleger ist dabei im übrigen nicht entscheidend, dass Grundstücke bislang als besonders sichere Anlagen galten. Wichtig ist vielmehr, dass sich der Anleger darauf verlassen kann, dass der Anteil an einem REIT oder einem Immobilienfonds nicht plötzlich viel riskanter wird. Denn solange der Grad des Risikos hinreichend verlässlich feststeht, kann der Anleger einen entsprechend höheren Ertrag verlangen. Darauf kann er sich aber nur einlassen, wenn er dieses Risiko in seinem Portfolio mit anderen, ebenso verlässlichen Risiken geeignet mischt ${ }^{14}$.

Bislang ist das Grundstücksrecht ganz anders gedacht. Die notarielle Form macht Grundstücksgeschäfte schwerfällig. Der öffentlich bestellte Notar soll den bisherigen Eigentümer fürsorglich vor der übereilten Verschleuderung seines Vermögens bewahren ${ }^{15}$. Die gleiche Sorge prägt das Vollstreckungsrecht. Das Bundesverfassungsgericht hat dafür gesorgt, dass Grundstücke in der Zwangsvollstreckung nicht unter der Hälfte ihres Verkehrswerts zugeschlagen werden dürfen ${ }^{16}$. Auf absehbare Zukunft wird es weiter Haushalte gegeben, deren wesentliches Vermögen aus dem Hausgrundstück besteht. Die Rechtsordnung darf die bisherigen Schutzvorschriften deshalb nicht aufgeben. Sie werden sogar ganz besonders gebraucht, wenn institutionelle Investoren beginnen, sich auch in diese Grundstücksmärkte einzukaufen. Weil die deutsche Gesellschaft schnell altert, wird es nicht wenig Menschen geben, die ihr Hausgrundstück bis zum Lebensende bewohnen, daraus aber ein zusätzliches Einkommen erzielen wollen. Es liegt nahe, dass institutionelle Anleger dafür Rentenverträge anbieten. Hier wird ein neues Problem des Verbraucherschutzes entstehen, dessen sich die Rechtsordnung annehmen sollte ${ }^{17}$. Es sollte nicht zu leicht sein, alte Menschen bei dieser Entscheidung zu übervorteilen, etwa durch Verfallklauseln, wenn sie die vertraglichen Pflichten zur Pflege der Immobilie nicht mehr wahrnehmen können.

14 Zum Hintergrund Jan Pieter Krahnen, Der Handel von Kreditrisiken: Eine neue Dimension des Kapitalmarktes, in: Perspektiven der Wirtschaftspolitik 6 (2005) 499-519.

15 BGH NJW 1990, $390 \mathrm{f}$.

16 BVerfGE 46, 325.

17 Zum geltenden Recht Schöner/Stöber Handbuch der Rechtspraxis Grundbuchrecht14, R 3236-R 3253. 
Für das Verhältnis institutioneller Anleger zueinander passt das bisherige Grundstücksrecht dagegen nicht. An der notariellen Form wird der Gesetzgeber vermutlich aus Gründen der Rechtsklarheit und des Verkehrsschutzes festhalten ${ }^{18}$. Aber der staatlichen Fürsorge bedürfen institutionelle Anleger nicht. Dagegen sind Rechtsregeln dringlich, die solche Personen schützen, die einen Teil ihres Vermögens in REIT-Anteilen investieren. Das aus dem Gesellschaftsrecht bekannte Problem des Auseinanderfallens von Herrschaft und Haftung erreicht nun auch das Grundstücksrecht ${ }^{19}$. Über die Anlagepolitik von REITs entscheiden angestellte Manager. Ihre Interessen müssen nicht mit denen der Anleger übereinstimmen ${ }^{20}$. Die Grundstücksfinanzierung wird durch REITs von Fremdkapital auf Eigenkapital umgestellt. Der REIT finanziert ein neues Objekt nicht durch einen Bankkredit, sondern durch die Ausgabe neuer Aktien. Der Effekt dieses Wandels ist von der Unternehmensfinanzierung bekannt. Der Fremdkapitalgeber partizipiert nicht an den Gewinnen. Deshalb ist zu besorgen, dass der Kapitalnehmer übermäßig riskante Geschäfte macht. Bei der Finanzierung durch Eigenkapital kann dagegen der Kapitalnehmer den Nutzen seiner Anstrengungen nicht in vollem Umfang behalten. Deshalb besteht die Gefahr, dass sich die Manager der REITs nicht genügend anstrengen. Den Nachteil hätten die Anleger ${ }^{21}$.

Dass Eigentum und Nutzung auseinanderfallen, ist an den Grundstücksmärkten an sich nicht ungewohnt. Die meisten Menschen wohnen zur Miete. Auch viele Unternehmen arbeiten in gemieteten Räumen. Bislang lag das vor allem daran, dass die Mieter die Anfangsinvestition nicht aufbringen konnten oder wollten. Viele Mieter schätzten auch die größere Flexibilität, etwa weil sie an ihrem Arbeitsmarkt mobil sein mussten. Vor allem bei gewerblich genutzten Immobilien spielte auch das Steuerrecht eine Rolle. Künftig tritt ein weiterer Grund hinzu. Im Leben eines Haushalts oder Unternehmens ist der Kauf eines Grundstücks ein seltenes Ereignis. Wer sich auf das Angebot von REITs verlässt, braucht die dafür erforderliche Expertise nicht mehr. Vielleicht werden bestimmte Arten von Grundstücken auf Dauer auch nur noch zwischen REITs gehandelt. Jedenfalls wird der Berufszweig weniger bedeutsam werden, der diese Expertise bislang zur Verfügung gestellt hat: die Grundstücksmakler.

In den USA sind große REITs besonders erfolgreich ${ }^{22}$. Es liegt nahe, dass deutsche REITs dem Modell folgen. Dann würde es auf die Dauer auch in Deutschland zur Konzentration von Grundstückseigentums in der Hand weniger institutioneller Anleger kommen. Daraus entsteht ein Machtproblem, dessen sich das Kartellrecht annehmen muss. Es wird vor allem darauf ankommen, den relevanten Markt angemessen zu definieren. Aus der Sicht der Anleger kommt es allein

18 Zu diesen Funktion von $\S 311$ b BGB s. BGHZ 53, 189, 195; 58, 386, 394; 87, 151, 153.

19 S. nur Michael C. Jensen und William H. Meckling, Theory of the Firm. Managerial Behaviour, Agency Cost and Ownership Structure, in: Journal of Financial Economics 3 (1976) 305-360.

20 Den empirischen Nachweis liefern für die USA Jay C. Hartzell, Libo Sun und Sheridan Titman, The Effect of Corporate Governance on Investment: Evidence from Real Estate Investment Trusts, in: Real Estate Economics 34 (2006) 343-376; s. auch Susanne Ethridge Cannon und Stephen C. Vogt, REITs and Their Management. An Analysis of Organizational Structure, Performance and Management Compensation, in: Journal of Real Estate Research 10 (1995) 297-317.

21 Näher Martin Hellwig, Die volkswirtschaftliche Bedeutung des Finanzsystems, in: Obst und Hintner (Hrsg.), Handbuch des Finanzsystems. Stuttgart (2000) 3-37.

22 Brent W. Ambrose, Michael J. Highfield und Peter D. Linneman, Real Estate and Economies of Scale. The Case of REITs, in: Real Estate Economics 33 (2005) 323-350. 
auf die Verlässlichkeit der Risikostruktur an. In dieser Dimensionen ist der sachlich und der räumlich relevante Markt folglich weit abzugrenzen. Ebenso verhält es sich beim Wettbewerb institutioneller Anleger um Anlageobjekte. In der vermutlich langen Übergangszeit geht es aber nicht bloß um den Wettbewerb zwischen institutionellen Anlegern. Aus der Sicht der nicht institutionellen Nachfrager nach Grundstücken sind viel engere sachliche und räumliche Märkte relevant. Das ist erst recht so aus der Sicht derer, die Gewerbeflächen oder Wohnraum nachfragen. In dieser Hinsicht ist der Markt deshalb viel enger abzugrenzen.

Wenn etwas ohnehin dem Staat gehört, dann braucht er keine Rechtsregeln, um öffentliche Interessen durchzusetzen ${ }^{23}$. Gerade im Grundstücksrecht hat der Staat in der Vergangenheit von dieser Möglichkeit Gebrauch gemacht. Ein Beispiel sind die zum Teil großen Wohnungsbestände der Kommunen. Sie sind offen in sozialpolitischer Absicht angelegt worden. Aus ähnlichen Gründen haben sich die Kommunen in Pflegeimmobilien engagiert ${ }^{24}$. Wenn die Kommunen solche Grundstücke an REITs verkaufen und die sozialpolitische Absicht fortbesteht, müsste die Kommune Einwirkungsmöglichkeiten behalten. Hat sie dafür aber überhaupt die Kompetenz? Wie sollte das praktisch geschehen, wenn der Investor Planungssicherheit verlangt?

Einen verwandten Effekt gab es in der Vergangenheit allein deshalb, weil Grundstücke demjenigen gehörten, der sie gegenwärtig nutzte. So gehören der Bahn Trassen und Bahnhofsgelände in begehrten Innenstadtlagen. Könnte man die Grundstücke zu anderen Zwecken nutzen, hätten sie ein Vielfaches ihres gegenwärtigen Werts. Bei vielen Traditionsgeschäften in den Innenstädten liegt es genauso. Ihr wertvollster Gegenstand ist das Grundstück. Würden die Eigentümer ihren Gewinn maximieren, würden sie das Geschäft schließen und das Grundstück verkaufen. Wären diese Grundstücke schon heute in der Hand von institutionellen Investoren, wäre dieser Wandel längst vollzogen. Die Investoren würden die marktübliche Miete verlangen. Wenn die bisherigen Nutzer diese Miete nicht aufbringen können, weichen sie in billigere Lagen aus oder schließen das Gewerbe. Auf diese Weise würden knappe Grundstücke produktiver verwendet. Dabei könnten aber solche öffentlichen Interessen leiden, die bislang reflektorisch durch die Trägheit der Grundstückseigentümer geschützt wurden. Auch stakeholder wären betroffen, insbesondere die jeweiligen Arbeitnehmer. Das ist kein Plädoyer dafür, solchen Wandel mit den Mitteln des Rechts zu verhindern. Wohl sollte die Rechtsordnung aber aufmerksam beobachten, welche schützenswerten Interessen auszufallen drohen, und gegebenenfalls alternative Lösungen finden.

23 Jean-Jacques Laffont und Jean Tirole, A Theory of Incentives in Procurement and Regulation. Cambridge, Mass. (1993); Christoph Engel, Die öffentliche Hand zwischen Innen- und Außensteuerung, in: Henneke (Hrsg.), Organisation kommunaler Aufgabenerfüllung. Stuttgart (1998) 145-222.

24 Klaus-R. Wagner, Zum Reit-Gesetzentwurf der Bundesregierung, in: Neue Zeitschrift für Gesellschaftsrecht (2006) 846-848,848. 


\section{Folgen für das Mietrecht}

Kaum ein Gewerbe ist in Deutschland so dicht reguliert wie die Vermietung von Wohnraum. Man sollte deshalb denken, dass sich die Rechtsordnung um den Mieterschutz auch dann keine Sorgen machen muss, wenn ein erheblicher Teil des Wohnungsbestands institutionellen Anlegern gehört. Professionelle Mietmanager mögen allerdings geschickter darin sein, die Lücken des Mieterschutzes zu finden und auszunutzen. Wem in einem Stadtteil fast alle Wohnungen gehören, der kann sich selbst seine Vergleichsmiete schreiben ${ }^{25}$. Er kann die Befugnis aus $\S 573$ II Nr. 3 BGB zur Verwertungskündigung nutzen ${ }^{26}$, Wohnungen luxuriös modernisieren oder gleich in Eigentumswohnungen umwandeln ${ }^{27}$. Die AGB-Kontrolle von Mietverträgen mag künftig eine größere Rolle spielen. Man kann sich fragen, ob der Grundsatz „Kauf bricht nicht Miete“ zum Schutz der Mieter noch ausreicht, oder ob man Regeln nach der Art der Vorschriften für die Bestandsübertragung von Versicherungsverhältnissen braucht ${ }^{28}$.

Schwerer wiegt eine andere Sorge. Um als Anlage attraktiv zu sein, müssen institutionelle Investoren dafür sorgen, dass das Risiko exakt definiert bleibt. Das schließt zwar, wie dargelegt, höhere Risiken nicht aus, solange ihnen eine höhere Rendite gegenübersteht. In der Konsequenz müssten Mieter mit einem ernst zunehmenden Mietausfallrisiko dann aber auch höhere Mieten zahlen. Noch wahrscheinlicher ist, dass die Manager die Vermietungsabteilungen anweisen, gleich nur solche Mieter zu akzeptieren, bei denen das Ausfallrisiko minimal ist. Dann würde am Wohnungsmarkt derselbe Effekt eintreten, der am Arbeitsmarkt offenkundig ist. Der Kündigungsschutz führt dazu, dass weniger attraktive Arbeitnehmer gar keinen Arbeitsplatz finden ${ }^{29}$. Ebenso würde der Mieterschutz dazu führen, dass nur noch Bewerber mit guter Bonität eine Wohnung finden. Genauso würde es Mietern ergehen, die in Verdacht geraten, den Hausfrieden zu gefährden.

In je weniger Händen die Wohnungsbestände konzentriert sind, desto stärker wird der Anreiz für die Mieter, durch Organisation Gegenmacht zu bilden. Daraus könnte sich auf mittlere Frist ein kollektives Mietrecht entwickeln, ähnlich dem kollektiven Arbeitsrecht. Dann würden Regeln gebraucht, die sagen, wer für wen sprechen kann, wer gebunden ist, welche Kampfmaßnahmen zulässig sind, und wie sich der Eigentümer wehren darf.

An sich ist die Vermietung von Wohnraum ein nationales Geschäft. Weil niemand an entfernten ausländischen Orten wohnen will, kann jedes Land ziemlich ungehindert seine eigene Wohnungspolitik treiben. Das Gewerbe ist vor Systemwettbewerb an sich wirksam geschützt ${ }^{30}$. Je stärker sich der deutsche Markt für institutionelle Anleger öffnet, desto mehr ändert sich das. Der

25 Bei gewerblich genutzten Gebäuden läuft die Vergleichsmiete aus diesem Grunde in manchen Kommunen schon heute weitgehend leer.

26 Eusani (FN 9), 74.

27 Ebd.,71.

28 Zu den verfassungsrechtlichen Vorgaben vgl. BVerfGE 114,1.

29 Näher Christoph Engel, Arbeitsmarkt und staatliche Lenkung, in: Veröffentlichungen der Vereinigung der Deutschen Staatsrechtslehrer 59 (2000) 56-98.

30 Fritz Wilhelm Scharpf, Regieren in Europa. Effektiv und Demokratisch ? Frankfurt (1999),14 spricht anschaulich von ,geschützten Sektoren“. 
Produktmarkt bleibt zwar national. Der Wettbewerbsdruck kommt aber von den vollständig internationalisierten Kapitalmärkten ${ }^{31}$. Die Renditeerwartungen der Anleger an den deutschen Grundstücksmärkten werden sich künftig deshalb an den Renditen für vergleichbar sichere Anlagen im Ausland orientieren.

Dieser Effekt lässt sich auch empirisch belegen. Es gibt zwar länderspezifische Ausschläge. Die Erträge der REITs hängen aber weltweit voneinander $a b^{32}$. Dabei hat der amerikanische Markt eine Führungsrolle. Veränderungen in den amerikanischen Märkten wirkten sich im Vereinigten Königreich sehr viel stärker aus als umgekehrt ${ }^{33}$. Diesen Druck wird das deutsche Mietrecht spüren. Man braucht kein Prophet zu sein, um die Deregulierung des Mietrechts vorherzusagen, sobald ein erheblicher Teil des Wohnungsbestands im Eigentum institutioneller Investoren steht. Denn die meisten Sicherungen machen den Bau von Mietwohnungen weniger ertragreich.

\section{Folgen für das Baurecht}

Wenn ein Industrieunternehmen einen neuen Standort plant, steht die Planungshoheit der Gemeinde nur auf dem Papier. Das Unternehmen verhandelt mit mehreren Gemeinden. Eine Gemeinde, die nicht bereit ist, ihre Bauplanung an den Wünschen des Unternehmens auszurichten, wird das Unternehmen nicht als neuen Standort wählen ${ }^{34}$. Wenn viele Grundstücke in der Gemeinde ein und demselben Investor gehören, werden auch die Bebauungspläne für fungible Gewerbeflächen und für Wohngebiete ausgehandelt werden. Die Konzentration des Eigentums in der Hand weniger verschafft diesen, politikwissenschaftlich gesprochen, eine Vetoposition ${ }^{35}$. Gegen ihren Willen kann die Gemeinde keine Politik mehr machen. Am Bauplanungsrecht zeigt sich besonders deutlich, wie wichtig die ordoliberale Konzeption des Kartellrechts ist. Die Ordoliberalen haben von Anfang an gefordert, dass das Recht nicht nur die Marktgegenseite, sondern auch die politischen Entscheidungsträger vor wirtschaftlicher Macht schützen solle ${ }^{36}$. Dem entspräche es, wenn man die einzelne Gemeinde als räumlich relevanten Markt definiert.

Nur ganz wenige Orte der Welt kommen ohne Bauleitplanung aus. Die berühmteste Ausnahme ist Houston Texas ${ }^{37}$. In Dörfern und Städten leben Menschen so dicht aufeinander, dass sich der Bedarf nach gegenseitiger Abstimmung nur schwer in Verträge auflösen lässt. Bei einer gänzlich

31 Zum Systemwettbewerb grundlegend Monopolkommission, Systemwettbewerb. Baden-Baden (1998); Markus Müller, Systemwettbewerb, Harmonisierung und Wettbewerbsverzerrung. Baden-Baden (2000).

32 Bond, Karolyi und Sanders (FN 4), 492 und passim.

33 David Michayluk, Patrick Wilson und Ralf Zurbruegg, Asymmetric Volatility, Correlation and Returns Dynamics Between the US and U:K: Securitized Real Estate Markets, in: Real Estate Economics 34 (2006) 109131.

34 Das baurechtliche Schrifttum hält sich meist bedeckt. Deutlich etwa Winfried Brohm, Beschleunigung der Verwaltungsverfahren - Straffung oder konsensuales Verwaltungshandeln? Zugleich ein Beitrag zu den Voraussetzungen der „Mediation“ in den USA und den strukturellen Unterschieden zwischen amerikanischem und deutschem Recht, in: Neue Zeitschrift für Verwaltungsrecht 10 (1991) 1025-1033, 1029.

35 Ellen M. Immergut, Health Politics. Interests and Institutions in Western Europe. Cambridge England ; New York, NY (1992); George Tsebelis, Veto Players. How Political Institutions Work. Princeton, N.J. (2002).

36 S. nur Franz Böhm, Wirtschaftsordnung und Staatsverfassung. Tübingen, (1950).

37 Einzelheiten bei Christopher Berry, Land Use Regulation and Residential Segregation. Does Zoning Matter?, in: American Law \& Economics Review 3 (2001) 251-274. 
dezentralen Lösung bleiben entweder viele an sich berechtigte Anliegen auf der Strecke. Oder es gäbe so viele Abwehrrechte, dass sich die Nachbarn gegenseitig blockieren ${ }^{38}$.

Der einzig denkbare privatrechtliche Ausweg spielte in der Vergangenheit in Deutschland nur eine untergeordnete Rolle. Ein Generalunternehmer kann einen ganzen Stadtteil bebauen. Durch Dienstbarkeiten sichert er die von ihm gewünschten Regeln des Zusammenlebens sachenrechtlich ab. Er verkauft die Grundstücke erst anschließend. Wenn der Großteil der Grundstücke im Eigentum weniger institutioneller Anleger steht, könnte diese Lösung dagegen häufiger werden. Dann rückt ein institutioneller Anleger in die Rolle eines privaten Regulierers. Das Verfassungsrecht muss sich auch aus diesem Grund mit der Rahmenordnung für private Regulierung befas$\operatorname{sen}^{39}$.

Außerdem muss das Verhältnis zwischen öffentlicher Bauplanung und privater Grundstücksordnung geklärt werden. Muss die Gemeinde die mit Dienstbarkeiten gesicherte Ordnung respektieren, wenn sie später den Bebauungsplan ändern will? Kommt es darauf an, ob die Grundstücke bei Erlass des Bebauungsplans noch (sämtlich?) in der Hand des institutionellen Anlegers sind? Dann könnte er die Dienstbarkeiten ja nach seiner Willkür jederzeit ändern. Hat die Gemeinde einen kompensierenden Anspruch darauf, auf die private Regulierung einzuwirken? Können Dritte verlangen, dass der private Regulierer ihre Anliegen ernst nimmt und in seine planerische Entscheidung einfließen lässt? Können auf diesem Wege auch öffentliche Interessen in die Entscheidung eingeführt werden, die nicht von der Gemeinde repräsentiert werden? Was geschieht also mit den normativen Anliegen, die bislang im Fachplanungsrecht ihren Ort haben?

\section{Der rechtliche Rahmen}

All das liest sich wie eine Geschichte von der Urgewalt der Kapitalmärkte, denen die deutsche Rechtsordnung wie ein Spielball ausgeliefert ist. Zumindest juristisch ist das nicht richtig. Im Mutterland der REITs waren sie eine Erfindung des Gesetzgebers. Er wollte es im Jahre 1960 für jedermann einfacher machen, in Grundstücken zu investieren ${ }^{40}$. Richtig sind die REITs in den USA erst gewachsen, nachdem ihnen 1986 auch gestattet wurde, die Grundstücke selbst zu managen $^{41}$. Ein weiteres Gesetz hat Grundstücksgesellschaften in anderer Rechts- und Organisationsform erlaubt, ihren Grundstücksbestand ohne schädliche Steuerwirkungen an REITs zu ver-

38 Christian Schubert, Die rechtliche Steuerung urbanen Wandels. Eine konstitutionenökonomische Untersuchung. Tübingen (2006); für das Phänomen hat Michael Heller den einprägsamen Begriff der ,,anticommons“ geprägt, Michael A. Heller, The Tragedy of the Anticommons. Property in the Transition from Marx to Markets, in: Harvard Law Review 111 (1998) 621-688.

39 Christoph Engel, A Constitutional Framework for Private Governance, in: German Law Journal 5 (2004) 197-236; Christoph Engel, Freiheit und Autonomie, in: Merten und Papier (Hrsg.), Handbuch der Grundrechte in Deutschland und Europa II. Heidelberg (2006) 389-436.

$40 \quad$ Capozza und Seguin (FN 3), 307.

41 Ebd. 
kaufen $^{42}$. Der Gesetzgeber hat deshalb sehr weitgehend in der Hand, welchen Einfluss REITs auf die deutschen Grundstücksmärkte bekommen.

Von dieser Möglichkeit hat der deutsche Gesetzgeber mit Wirkung vom 1.1.2007 Gebrauch gemacht $^{43}$. Der entscheidende Anreiz ist die in $\S 16$ des Gesetzes ausgesprochene Befreiung von der Körperschafts- und der Gewerbesteuer. Ursprünglich sollten Wohnungsimmobilien unbegrenzt einbezogen werden ${ }^{44}$. Nun gilt das Gesetz nach seinem $\S 1$ I Nr. 1 nicht für „Bestandsmietwohnimmobilien“. In $\S 3$ IX findet sich die folgende Legaldefinition:

„Bestandsmietwohnimmobilien sind Immobilien, die überwiegend Wohnzwecken dienen, sofern diese vor dem 1.Januar 2007 erbaut worden sind“".

Mietwohnungen werden also erst schrittweise in die neue Eigentumsstruktur überführt. Auch eine weitere Regel ist als Instrument zum Mieterschutz gemeint ${ }^{45}$. Nach $\S 11$ I müssen sich mindestens $15 \%$ der Aktien im Streubesitz befinden. Schließlich schreibt $\S 14$ I vor, dass der REIT innerhalb von fünf Jahren maximal die Hälfte seines durchschnittlichen Immobilienbestands veräußern darf. Nach den amerikanischen Erfahrungen wird auch diese Regel bremsend wirken.

Also viel Lärm um Nichts? Wohl kaum. Nichts hindert den Gesetzgeber, die bremsenden Regeln abzuschaffen, wenn er REITs mehr Entwicklungsraum geben will. Auch in den USA sind diese Hindernisse erst im Laufe der Zeit abgeschafft worden. Vor allem wird der deutsche Gesetzgeber aber dem Druck der Kapitalmärkte ausgesetzt bleiben. Dabei spielt nicht nur die Sorge eine Rolle, Deutschland könnte Wachstumschancen verpassen. Vielmehr verhindert das geltende Recht nicht, dass ausländische institutionelle Investoren deutsche Grundstücke erwerben ${ }^{46}$. Dafür gibt es schon Beispiele. Auch ausländische REITs haben sich an den deutschen Grundstücksmärkten engagiert ${ }^{47}$. Der deutsche Gesetzgeber könnte sich schon bald gedrängt sehen, diesem Ausverkauf deutschen Bodens durch liberalere Regeln für deutsche REITs vorzubeugen. Auf beiden Wegen wird das geltende deutsche Recht also dem Druck des Systemwettbewerbs ausgesetzt sein. Selbst wenn nichts davon geschehen sollte, würde der aufgezeigte Wandel aber stattfinden. Er braucht dann nur mehr Zeit.

42 Ebd., 308.

43 Gesetz über deutsche Immobilien-Aktiengesellschaften mit börsennotierten Anteilen (REIT-Gesetz) vom 28.5.2007, BGB1. I 914.

$44 \quad$ Eusani (FN 9), 71.

45 Jürgen van Kann, Clemens Just und Joachim Krämer, Der Regierungsentwurf eines Gesetzes zur Schaffung deutscher Immobilien-Aktiengesellschaften mit börsennotierten Anteilen (REIT-Gesetz), in: Deutsches Steuerrecht (2006) 2105-2111, 2106.

46 Eusani (FN 9), 72.

47 Schacht und Gänsler (FN 1), 105. 